\title{
HIGH-CURRENT MICRO-PULSE ELECTRON GUNS AND ACCELERATOR APPLICATIONS*
}

\author{
S. K. Guharay, L. K. Len and F. Mako, FM Technologies, Inc., Fairfax, VA 22039, USA
}

\begin{abstract}
A major research activity is being pursued at FMT to develop high-current, short-duration pulses of electron beams for injectors in electron accelerators as well as for microwave generation in klystrons and related devices. Design and development of gun cavities, in the frequency range of 1 to $12 \mathrm{GHz}$, are conducted through a comprehensive task including simulation, analytical and experimental studies. With the goal to build high-power, high-frequency klystrons, one of our most recent efforts is focused on developing an X-band cavity at about $9 \mathrm{GHz}$ for generating high-current, bunched micropulses of electron beams. The full system has been designed and built, and it is running reliably. An average beam current density $>20 \mathrm{~A} / \mathrm{cm}^{2}$ over a macropulse, or $400 \mathrm{~A} / \mathrm{cm}^{2}$ over a micropulse, has been measured for a modest input rfpower, $\sim 60 \mathrm{~kW}$, into the gun cavity. In addition, experiments are being conducted to develop an electron gun at $1.3 \mathrm{GHz}$, with gating capabilities, and match beam requirements for Argonne Wakefield Accelerator.
\end{abstract}

\section{INTRODUCTION}

The development of high-current, short-duration pulses of electron beams has posed challenging problems. Highcurrent pulses are demanded in injector systems for electron accelerators, both for industrial linacs and for high energy accelerators for linear colliders. Shortduration pulses are used for microwave generation in klystrons and related devices. Several groups [1-7] have been working on microwave generation, especially in view of the requirements of future $\mathrm{TeV}$ linear colliders. A major research and development activity is being conducted at FMT, and robust micropulse electron guns (MPG) have been developed $[8,9]$ to produce selfbunched, high-current-density beams. The natural bunch formation in this cavity is a phase locking resonant process which is not prone to phase instability. Furthermore, unlike thermionic or field emission devices, which have a relatively short life time, the mechanism of MPG does not cause erosion or evaporation, and therefore, it has distinct merits for providing long life time. FMT designed and built a prototype L-band (1.3 $\mathrm{GHz})$ and S-band $(2.85 \mathrm{GHz})$ MPG. The L-band MPG was designed for and produced $\sim 1.1 \mathrm{nC}$ per microbunch and ran successfully for 1.5 years at 24 hours a day. This article describes the development of an X-band gun for a micropulse klystron (MPK). Work on S- and L-bands were described earlier [8,9]; updates on current activities on L-band gun are discussed here briefly. This MPK gun will provide a high-power, high frequency source for many applications including high energy accelerators for linear colliders, free electron lasers, medical and industrial rf linacs.

While our goal is to build an rf-gun in $\mathrm{TM}_{020}$ mode, our initial efforts were focused to operating the cavity in $\mathrm{TM}_{010}$ mode and obtain valuable guidelines for efficiently setting up the $\mathrm{TM}_{020}$ mode. Design issues of the X-band gun cavity and experimental results are described in Sec. 2. Our activities on L-band gun are briefly stated in Sec.3. Finally conclusions are given in Sec. 4.

\section{HIGH-CURRENT, SHORT PULSE X- BAND ELECTRON GUN - SIMULATION AND EXPERIMENTS}

\subsection{Test Cavity for Benchmarking GdfidL Code Predictions:}

Our past experience with simulation predictions by GdfidL prompted us to adapt this code to design the rfcavity for an X-band MPK gun. Following the mechanisms for operation of this class of rf-guns, pioneered at FMT, it was derived that the saturation current density from the gun maximizes when a characteristic parameter, $\alpha_{0}=\frac{e V_{0}}{m \omega^{2} d^{2}}$, has a value of 0.373 . Note that this parameter includes peak rf-voltage $\mathrm{V}_{0}$ in the cavity, $\mathrm{rf}$ frequency $\omega$ and electrode gap $\mathrm{d}$. Guided by this criterion we pursued simulation studies and determined optimum cavity characteristics.

The resonant frequency of the cavity is determined by its volume confining the $\mathrm{rf}$ field. The change of resonant frequency is governed by the relation $\frac{\Delta \omega}{\omega} \propto-\Delta V$. Here, $\Delta \omega$ is the frequency shift due to a perturbation in the volume of the cavity by $\Delta \mathrm{V}$. The negative sign indicates that the resonant frequency decreases with increasing $\mathrm{V}$. This feature is implemented by moving the electrode up (when $\Delta \mathrm{V}$ is positive) or down (when $\Delta \mathrm{V}$ is negative) into the cavity and thereby, the resonant frequency is varied. Another practical "knob" for frequency adjustment is the distance between sidewalls of the gun cavity. These sidewalls pertain to faces perpendicular to the direction of propagation of the input $\mathrm{rf}$ field and also to the gap between electrodes in the cavity. Typically two copper side plungers are placed sideways facing each other. It is important to insure good rf-contact between the walls of the side plunger and the cavity body. The distance

\footnotetext{
* Work supported by the U.S. Department of Energy under SBIR
} 
between the side plungers in the cavity is adjusted by moving the plungers with linear micrometers.

We performed detailed simulation studies to obtain interdependence between characteristic parameters for resonance conditions. Simulation studies guided us to choose a cavity height of $3.4 \mathrm{~mm}$ (this can be the maximum gap between the electrodes) and electrode radius of $2.56 \mathrm{~mm}$. The simulation results were critically examined by experimental measurements using a test cavity operated in cold mode (for very low rf-power, $\sim 10$ $\mathrm{dbm}$ ). Figure1 shows a comparison of simulation and experimental results. Here, the basic criterion is set to achieve cavity resonance at $9 \mathrm{GHz}$. Note that the simulation and experimental results agree reasonably well.

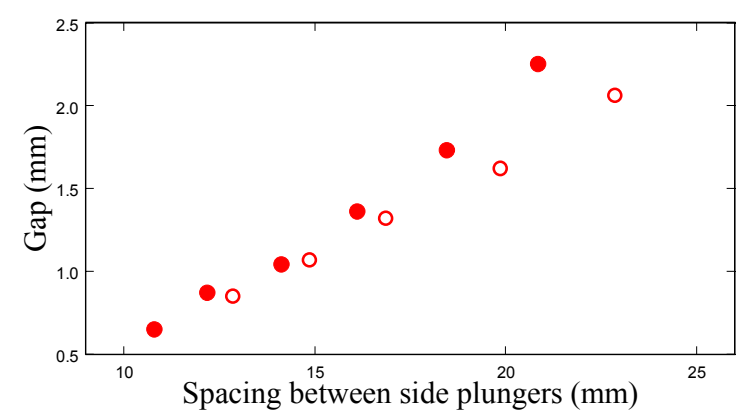

Figure 1 Variation of electrode gap with spacing between side plungers in a gun cavity for resonance at $9 \mathrm{GHz}---$ simulation results (open circles), measured data (solid circles).

\subsection{A Prototype MPK}

Above test experiments provided us confidence on simulation predictions, and we proceeded to build a prototype unit for its full-scale evaluation. Figure 2 shows

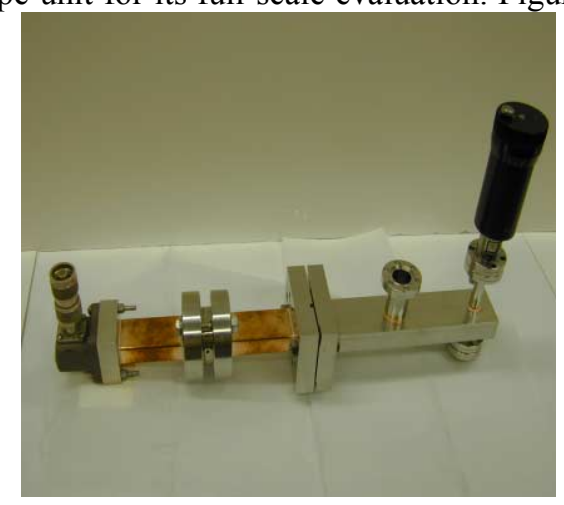

Figure 2 Photograph of X-band gun cavity

a photograph of the X-band cavity system. This highly compact unit includes a gun cavity with a moveable electrode mounted on a micrometer, a tapered waveguide section for efficient rf-power feed from a standard WR-90 waveguide into the gun cavity. All materials are 304L stainless steel. The cavity components including ports for various feedthroughs and vacuum were brazed together. This unit is pumped down to a base pressure of less than
$10^{-8}$ Torr using an ion pump. An rf-window, made of alumina, separates the vacuum section of the cavity from the upstream rf-input section. The upstream section beyond the end of the taper comprises a cross-guide coupler for measurements of forward and reflected rfpower, an isolator for isolation of reflected power into the magnetron (the source for input $\mathrm{rf}$ power) and appropriate bends for required mechanical couplings to the output of the magnetron. A tunable magnetron source and its associated electronics have been assembled. An output power of about $200 \mathrm{~kW}$ can be obtained from this source.

Note that no side plungers were included in this unit for simplicity. With a fixed spacing of $1.4 \mathrm{~cm}$ between the sidewalls of the gun cavity, the tuning of the cavity was accomplished by (a) varying the gap between the electrodes and (b) adjusting the frequency of the source for input rf power. We noticed that the gun cavity can be operated over the full tuning range of the source frequency, i.e., over 8.6 to $9.6 \mathrm{GHz}$, if the electrode gap is varied from about $0.75 \mathrm{~mm}$ to $1.8 \mathrm{~mm}$.

The prototype system discussed above has been running flawlessly. A typical beam macropulse is shown in Fig.3. The beam current through a hole of diameter $=1.4 \mathrm{~mm}$ was monitored by a collector. The collector was biased to suppress any secondary electrons from it. The beam pulse follows the input rf-pulse very well. It shows a good flat top during about $2 \mu$ s with negligibly small noise. The results are highly reproducible.

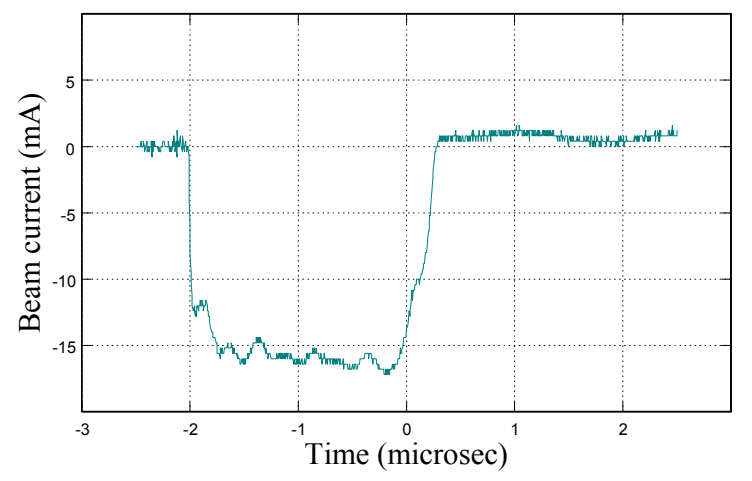

Figure 3 Typical beam macropulse

A systematic set of measurements was performed to obtain the variation of beam current density with rf-power in the cavity. Shown in Fig. 4 are measurements for three different gaps between the electrodes. The rf-power in the cavity was estimated as the difference between the forward and reflected if power measured at the crossguide coupler.

Detailed simulation results showed the saturated current density $\mathrm{J}_{\mathrm{s}}$ to scale as $\omega^{3}$ and $\mathrm{d}^{1.75}$, resonant peak voltage $\mathrm{V}_{0}$ in the cavity to scale as $\omega^{2}$, and peak particle energy to scale as $\mathrm{V}_{0}{ }^{1.012}$. Since no indications of any breakdown in the cavity were observed during the measurements in Fig. 4, much higher beam current densities can be obtained by increasing the input $\mathrm{rf}$ power. The scaling relationships suggest that larger gaps are desired to sustain a high saturated current density. 


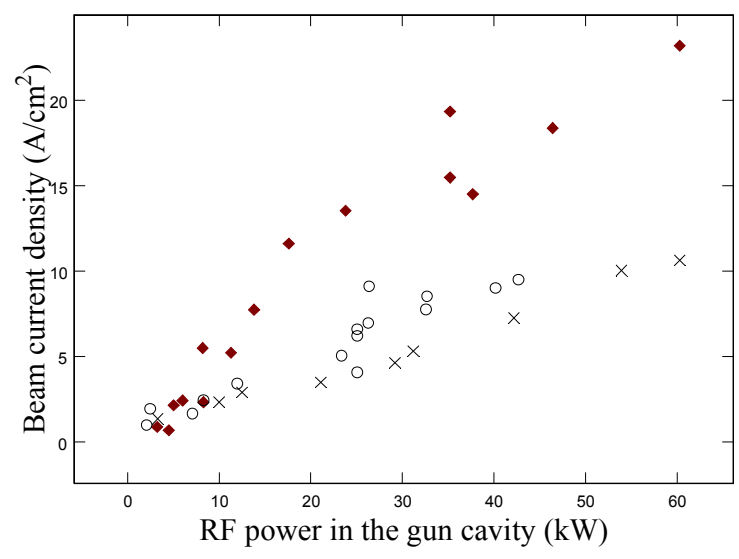

Figure 4 Variation of beam current density at the gun cavity exit with rf power for electrode gaps $d=1.82 \mathrm{~mm}$ (solid diamond), $1.51 \mathrm{~mm}$ (open circle) amd $1.1 \mathrm{~mm}$ (cross). Corresponding resonant frequencies were, respectively, $9.64 \mathrm{GHz}, 8.92 \mathrm{GHz}$ and $8.48 \mathrm{GHz}$.

\section{HIGH-CURRENT, GATED L-BAND ELECTRON GUN}

The above exercise has been pursued in the context of developing a high-current, gated electron gun for operation at $1.3 \mathrm{GHz}$. Simulation studies with GdfidL provided us the characteristic geometrical parameters of the gun cavity. Figure 5 shows a representative parameter space to achieve a resonant frequency of $1.3 \mathrm{GHz}$. The ability to operate at different gap spacings at fixed frequency enables the device to have current density adjustability. We built a test cavity and validated these simulation predictions. These studies guided us to build a prototype unit including the gun cavity and an appropriate tapered waveguide section. The photograph in Fig. 6 shows the gun cavity. Experiments with the prototype unit are in progress.

\section{CONCLUSIONS}

A comprehensive task encompassing simulation, analytical and experimental studies has been successfully launched to design and build rf-guns with the goal to reliably extract high current density, short pulse electron beams with low beam emittance. A prototype unit for operation in the X-band at $9 \mathrm{GHz}$ has been built and tested. $\mathrm{TM}_{010}$ modes are presently excited in the gun cavity. Average beam current densities $>20 \mathrm{~A} / \mathrm{cm}^{2}$ over a macropulse, or $>400 \mathrm{~A} / \mathrm{cm}^{2}$ over a micropulse, have been obtained at the exit of the gun cavity for an rf power of about $60 \mathrm{~kW}$. Much higher current densities can be achieved by increasing the input rf-power and further reducing losses due to reflections from the rf-window and perhaps from the iris in the gun cavity. With very valuable knowledge from the experiments with $\mathrm{TM}_{010}$ modes we built a test cavity for $\mathrm{TM}_{020}$ mode, and experiments are in

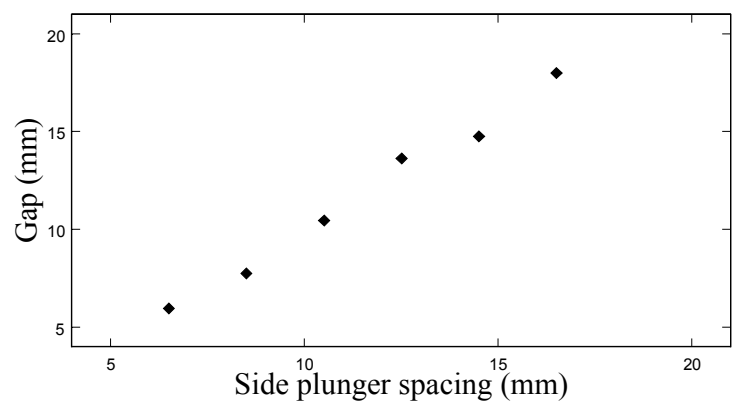

Figure 5 Variation of electrode gap with spacing between side plungers in an L-band cavity. The cavity height (maximum electrode gap) is $3 \mathrm{~cm}$.

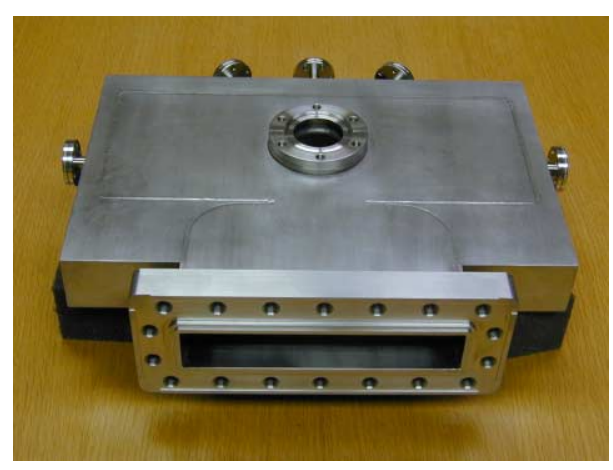

Figure 6 Photograph of L-band gun cavity

progress. Additionally we designed and built a prototype gun cavity system for operation in L-band at $1.3 \mathrm{GHz}$. Gating of the beam pulse is an additional unique feature which will be attributed to the present system. Detailed measurements of the beam characteristics and performance of the L-band cavity will be reported elsewhere.

\section{REFERENCES}

[1] V. L. Granatstein, et al., "High-power microwave sources for advanced accelerators", AIP Conf. Proc. No. 253, 1991.

[2] W. Lawson, et al., Phys. Rev. Lett. 67, 520 (1991).

[3] W. Lawson, et al., IEEE Trans. Plasma Sci. 1992.

[4] S. Tantawi, et al., IEEE Trans. Plasma Sci. (1992).

[5] Karlirner, et al., Nucl. Instrum. Meth. A269, 459 (1988).

[6] W. M. Manheimer, IEEE Trans. Plasma Sci. 18, 632 (1990).

[7] B. Hafizi, et al., IEEE Trans. Plasma Sci. 20, 232 (1992).

[8] F. Mako and W. Peter, Proc. Particle Accelerator Conf. IEEE Cat 93CH3279-1, 2702 (1993).

[9] L. K. Len and F. Mako, Proc. Particle Accelerator Conf. New York, 1999, p.70. 